\title{
Reactions to blatantly contradictory information
}

\author{
ELIZABETH F. LOFTUS \\ University of Washington, Seattle, Washington 98195
}

\begin{abstract}
In two experiments, subjects were shown a complex event and were later exposed to misinformation about that event. In addition, some subjects received a piece of blatantly contradictory misinformation. Blatant misinformation both was rejected by subjects and caused them to be more resistant to other misinformation that they would ordinarily have been inclined to accept (the "spillover" effect). However, delaying the blatant misinformation until the other pieces of false information had already been processed destroyed its ability to make subjects immune to such "ordinary" misinformation. These results are consistent with the idea that subjects incorporate new information into memory at the time it is initially introduced and use this information to update an existing memorial representation.
\end{abstract}

Between the time an event is observed and the time it is later recounted to someone else, a person can be exposed to new information about that event. This new information can become incorporated into the person's memory, supplementing or even altering what was previously acquired. A major theoretical question is how the new information is integrated into memory. Does the integration occur at the moment the new information is introduced? Or does it take place later, when the person is finally asked to recollect the original experience? The present experiments bear directly on this issue. The type of integration studied here has been observed in a number of previous experiments. For example, in one study subjects saw a filmed automobile accident and were later exposed to a piece of false information, namely, that a barn had appeared in the film. One week later they were asked whether they had seen the nonexistent barn. Over $17 \%$ of the subjects indicated that they had seen the barn when its existence had been suggested, while less than $3 \%$ indicated they had seen the nonexistent object when it had not been mentioned (Loftus, 1975). Apparently the "barn" misinformation was integrated into the subjects' memory of the event, thereby supplementing that representation. In other studies, it has been shown that new information can alter or transform a recollection. For example, in one study (Loftus, Miller, \& Burns, 1978) subjects saw a series of slides depicting successive stages in an auto. pedestrian accident and were then told that a traffic sign had been seen in the slides (e.g., some subjects

This research was supported by a grant from the National Science Foundation to Elizabeth Loftus. The writing of the manuscript was facilitated by grants from the National Science Foundation and the Andrew Mellon Foundation to the Center for Advanced Study in the Behavioral Sciences, where the author enjoyed the privileges of fellowship during 1978-1979. Thanks are due to Kathy Stotts and Peter Powers for the efforts they put into this research. Please address all correspondence to Elizabeth Loftus, Center for Advanced Study in the Behavioral Sciences, Stanford, California 94305. saw a stop sign and later received the suggestion that it was a yield sign). Under certain conditions, as many as $80 \%$ of the subjects indicated having seen a slide with that piece of misinformation. Again, the new "sign" information was apparently integrated into the subjects' memorial representation of the event, possibly altering that representation.

One purpose of the present research was to explore what happens when a person receives information that is implausible or even blatantly contradictory to what was actually seen. Is there a limit to the kinds of subsequent information that will appear in a witness's report? Put another way, in order for an introduced item to be incorporated into a person's recollection, must that item be plausible in light of the event that was witnessed? Or could a person be convinced that he or she had seen something as unlikely as, say, a skeleton dangling from a chandelier?

In a pilot study performed by K. Stotts, subjects viewed a set of unrelated slides. After viewing the slides, subjects were given a description of the slides that was ostensibly produced by a college professor who had viewed them three times longer than had the subjects. The professor's description contained both nonexistent items that were plausible within the context and nonexistent items that were implausible. For example, some subjects who had seen a slide of a city scene read a description with a telephone booth (plausible), while others read about a water pump (implausible). For counterbalancing purposes, still other subjects read about a telephone booth in a previously viewed farm scene (implausible) or about a water pump in that same scene (plausible).

After reading the "professor's" descriptions, subjects performed a recognition test. They indicated whether they had seen certain items in certain scenes and their confidence in their answer. They were urged to say "Yes" only to items they remembered seeing.

Subjects said "Yes" to having seen plausible nonexistent objects much more often than to implausible 
ones. In addition, implausible items used in this study were almost never recognized when they were not mentioned, but when mentioned the likelihood that a subject reported he or she had seen them increased substantially. Subjects claimed to have seen an implausible item $1 \%$ of the time when it was not mentioned in the professor's description but $21 \%$ when the item was mentioned. The corresponding percentages for the piausible items were 24 and 49 .

Finally, subjects were more confident in their responses about implausible items than plausible ones. Furthermore, they were more confident when they said "Yes" (an incorrect response) than when they said "No" (a correct response). Subjects were most confident when they reported they had seen an implausible item, indicating they would fall for an implausible item, but they required more confidence to do so. More simply put, you have to be pretty sure before you are willing to say you have seen a skeleton dangling from a chandelier.

While Stotts' implausible items were unlikely in the context of the slide to which they were attached, for the most part they did not contradict any specific detail. Thus, the manipulation left unanswered the question of what would happen if one introduced a piece of false information that blatantly contradicted a clearly perceived detail. One purpose of the present research was to explore this issue. A second was to see if the presence of patently false information would influence a person's ability to resist other more subtle suggestions (a "spillover" effect). This could occur if the blatantly contradictory detail caused people to scrutinize more carefully the rest of the information, leading to a greater resistance to the more subtle misleading suggestions. Alternately, any spillover effects of the blatant information could be occurring later, at the time the person's recollection is queried. When tested, both the original information and a piece of subtle misinformation are retrieved from memory. However, the blatant misinformation causes the person to disregard the subtle information that was conveyed with it The person then gives greater weight to his or her original memory.

This reasoning leads to what is perhaps the central purpose of the present research, namely, to shed light on how new information is integrated with existing knowledge. Does it occur at the moment the new information is introduced? Or does the integration take place later, when the person is finally asked to recollect the original experience? Our technique in Experiment 2 for exploring this issue was to present misinformation to people in two states. First, some subtle pieces of misinformation were presented; then, after some delay, a piece of blatantly false information was presented. If the subtle misinformation is incorporated into memory at the time it occurs, then the delayed blatantly false information might still be rejected, but it should have no spillover effect on the subject's memory for the other items. On the other hand, if the delayed blatantly false information has its effects at the time the subject is later tested, then delaying it should produce the same effect as introducing it early along with the other pieces of misinformation.

\section{EXPERIMENT 1}

\section{Method}

Subjects. Forty-six persons, mostly females, participated in this study, 32 for course credit and 14 for pay. The subjects were tested in groups ranging from two to six. Any subject who reported a problem with color blindness was given course credit and excused from the study

Procedure. Subjects were told that they would see a series of slides and would be asked a few questions about the slides. The overall procedure had four major phases: viewing the slides, filling out an "accuracy" questionnaire, reading a narrative, and taking a final test.

The slides. A sequence of 24 color slides depicting a walletsnatching incident in a small town was shown to the subjects. Each slide was presented for 5 sec.

The slide sequence opens with a young woman walking down a busy street. She meets a friend and stops to talk for a moment. As the woman continues down the street, she is approached by a man wearing a cowboy hat who bumps into her, causing her to drop her shopping bag. The man and woman both stoop to pick up some articles that had fallen out. When the woman is looking the other way, the man reaches into her shoulder bag and takes her wallet. The woman does not notice and the two part. Soon the victim becomes aware that her red wallet is missing, at which point two other women cross the street toward her and gesture in the direction of the fleeing man. One slide from the series is shown in Figure 1.

The accuracy questionnaire. After a short filler activity in which they provided names for various colors in a color chart, the subjects filled out a questionnaire designed to assess their memory for the slides. The questionnaire included 30 items addressing diverse details of the wallet-snatching incident. It asked about major details concerning the central characters, their clothing, and actions and about minor details, such as the surrounding environment, extraneous people, buildings, and iraffic. A separate group of 100 undergraduate volunteer sub. jects was used to test the reliability of items on this questionnaire. Questions found to have poor reliability $(<.60)$ were successively revised, with new items added to create the final 30-item list. The 30 items were declarative sentences with a missing pinrase or word and five alternatives. For example, one question was "The victim's friend was carrying

(a) a newspaper, (b) a shopping bag, (c) a notebook, (d) an umbrella, (e) none of the above." For each item, the subjects indicated their confidence in their answers using a 3-point rating scale, where 1 indicated that the subject was guessing and 3 indicated high confidence. After the questionnaire was completed, a filler activity was performed (pilot data for a colleague's unrelated experiment), and subjects were asked to return the following day.

The narrative. At the beginning of the second session, all subjects read a 1-page narrative containing a version of the incident that was allegedly written by a psychology professot who had seen the slides for $30 \mathrm{sec}$ each. The subjects were given no information about the factualness of the "professor's" description or the professor's general credibility. To conceal the purpose of this task, the subjects were asked to rate the narrative on several attributes, such as clarity of writing. Embedded within the full-page narrative were erroneous descriptions of four critical items. These contained some erroneous information 


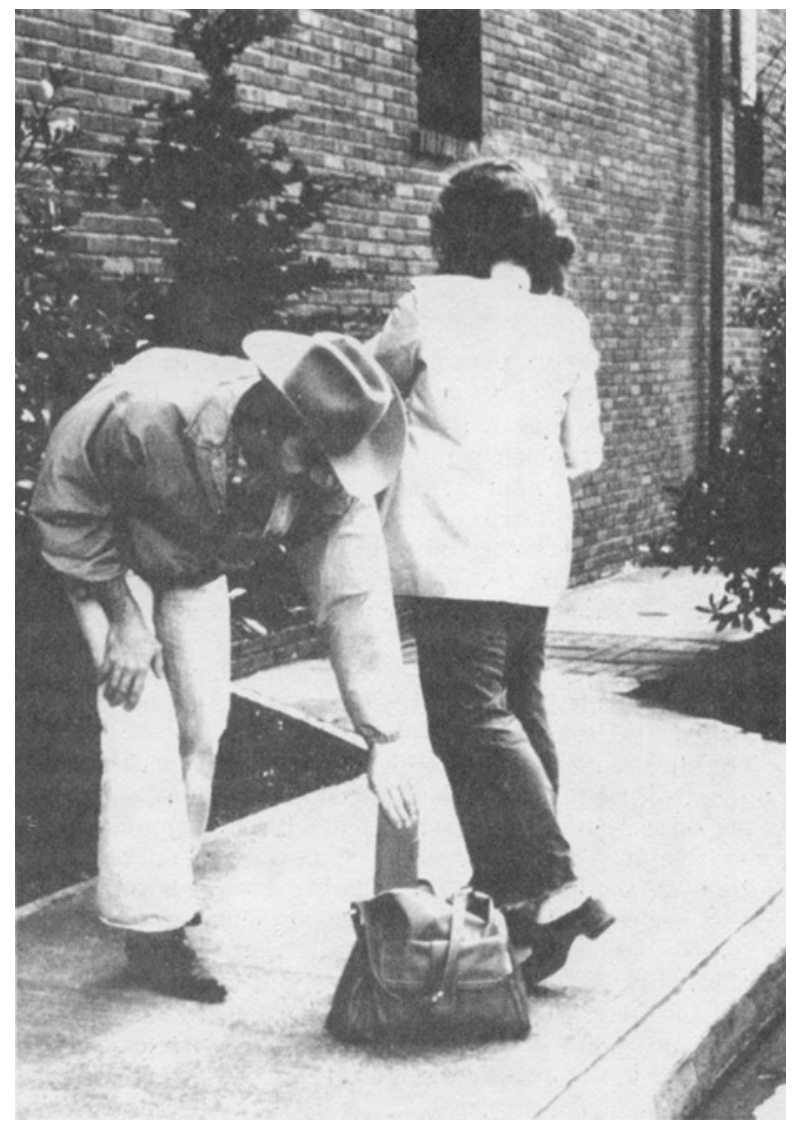

Figure 1. Black-and-white copy of 1 of 24 slides shown to experimental subjects.

about objects that were relatively peripheral to the central characters and action: a shirt displayed in a window, an overhanging sign, persons passing the victim, the object carried by the victim's friend.

In one version, the narrative contained, in addition, erroneous mention of a very obvious object. It referred to the red wallet taken by the thief as dark brown. We knew this detail was "blatantly" false because virtually everyone in a previous experiment (Powers, Andriks, \& Loftus, 1979) correctly identified its color, and $98 \%$ of the subjects in this experiment correctly identified its color on the accuracy questionnaire. One half of the subjects (blatant group) read a narrative containing the four key erroneous items plus the blatant item, whereas the other half of the subjects (the subtle group) read a narrative containing only the four key erroneous items. Subjects were randomly assigned to one of the two conditions.

After rating the narrative, the subjects completed a $10-\mathrm{min}$ filler activity and then were given a final test on the details of the slides.

The final test. The final test contained 20 items. The items were declarative sentences with three choices for a missing word or phrase. Subjects indicated their confidence using the same 3-point scale used in the accuracy test. They were urged to respond only on the basis of their own-not the professor'srecollection of the incident. The items on this test were generally different from those on the accuracy test. However, there was partial overlap in terms of the critical items. For example, the accuracy test queried about the type of item carried by the victim's friend (it happened to be a notebook), while the final test asked about its color.

\section{Results}

The major result from this experiment is that subjects given the blatant piece of misinformation uniformly rejected it. On a final test question asking about the color of the victim's wallet, all but two subjects correctly indicated that the color was red (with one choosing the suggested answer, "dark brown," and the other, some unrelated color). In addition, presence of a piece of blatant misinformation resulted in subjects' being more resistant to suggestions about the four subtle items. Their final test responses to the critical items were much more accurate.

The data analysis substantiating these conclusions is somewhat complicated. First, a subject's "accuracy" response on each of the four key items was converted to a numerical value. A correct response with a very high confidence rating received a 6 , a correct response with a moderate confidence rating received a 5 , a correct response with the lowest confidence rating received a 4 , an incorrect response with low confidence a 3, an incorrect response with moderate confidence a 2 , and an incorrect response with a high confidence rating a 1. Thus, a 6-point scale emerged which ranged from very low accuracy (1) to very high accuracy (6). In some sense this score reflected the appropriateness of confidence in an item.

Responses to the four subtle items on the final test were similarly converted to numerical values. For example, a score of 6 was assigned to a correct response given with high confidence. Such a score indicated that the subject persisted in recalling a correct detail-and did so with high confidence-despite the presence of a misleading suggestion. Thus, the subject showed very high "suggestibility resistance."

We wished to obtain a suggestibility resistance score corresponding to each of the six levels of accuracy; however, this was not a simple thing to do. Each subject provided responses to only four critical items. Accuracy on those items could range from 1 to 6 , as did the corresponding suggestibility resistance scores. Thus, at most, a subject provided data corresponding to four different accuracy scores. For example, one subject provided the accuracy scores 1, 2, 4, and 6. Often, however, subjects provided data corresponding to only two or three different accuracy scores. For example, one subject provided the accuracy scores 2, 2, 6, and 6 . The corresponding suggestibility resistance scores for those four items were $1,2,4$, and 6 , respectively. For this subject, we used a mean suggestibility resistance score of 1.5 to correspond to the accuracy score of 2 and 5 to correspond to the accuracy score of 6 . For this subject, it is evident that low accuracy corresponds to low resistance, high accuracy to high resistance.

When the individual subject scores had been recorded for each level of accuracy, we calculated a mean 
suggestibility resistance score by taking the mean of the scores provided by individual subjects. Figure 2 shows the relationship between accuracy on a particular item and mean suggestibility resistance for that item. The relationship is displayed separately for the blatant and subtle subjects.

Because each subject contributed to, at most, four points in Figure 2, it was not possible to analyze the data in this form. Thus, two suggestibility resistance scores were obtained for each subject, one when accuracy was low (i.e., the subject's answer was wrong) and the other when accuracy was high (the subject's answer was right). A response was considered to be accurate for purposes of this analysis if the subject answered it correctly, whatever his confidence level. Two mean suggestibility resistance scores were then obtained by taking averages across subjects. The means are shown in Figure 3.

A two-way analysis of variance was performed on these data and revealed two significant main effects and a significant interaction. Subjects were better able to resist a suggestion about an item if they had initially been accurate on that item than if they had not $[F(1,44)=44.07, \mathrm{MSe}=1.22, \mathrm{p}<.01]$. Subjects who were given blatant information resisted more suggestions than those who were not $[\mathrm{F}(1,44)=8.75$, MSe $=2.31, \mathrm{p}<.01]$. Finally, a blatant subject's advantage over his subtle counterpart in ability to resist suggestion was greater on an initially accurate item than on an initially inaccurate one $[\mathrm{F}(1,44)=6.48, \mathrm{MSe}=1.22$, $\mathrm{p}<.05]$. In fact, post hoc comparisons indicated that the blatant subjects were better able to resist suggestion when initially accurate but not when initially inaccurate. Nonparametric analysis led to the identical conclusions. It should also be mentioned that the blatant and subtle

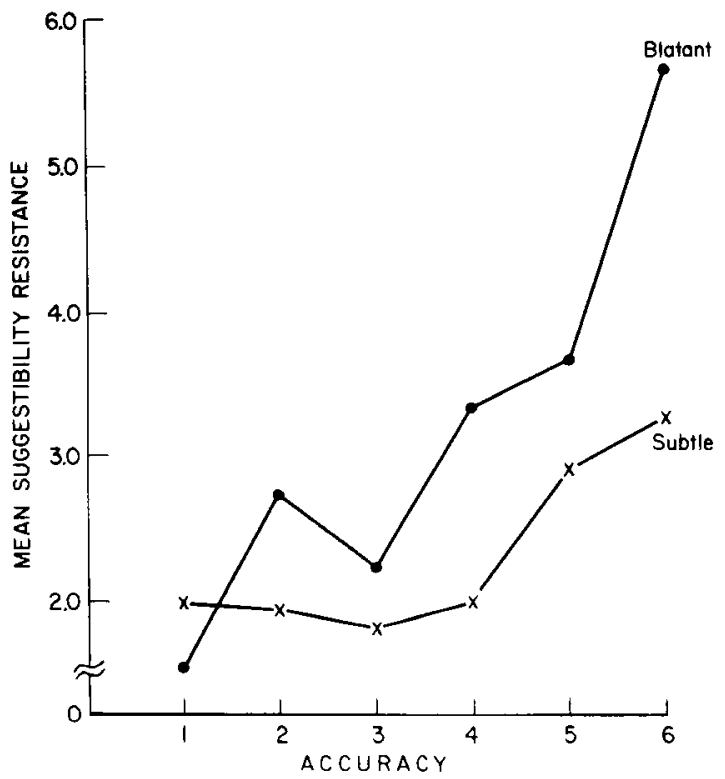

Figure 2. The relationship between accuracy and suggestibility resistance. Higher scores indicate a greater ability to resist a misleading suggestion.

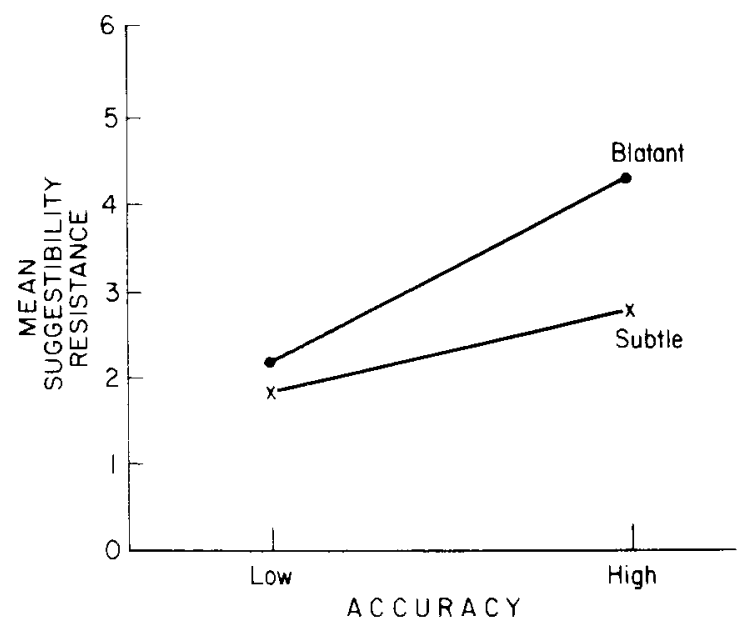

Figure 3. Mean suggestibility resistance on an item as a function of initial accuracy on that item, presented separately for subjects given a blatant suggestion and those given a subtle suggestion.

subjects did not differ in terms of initial accuracy $(\mathrm{t}<1)$.

Level of confidence. For the blatant and subtle subjects combined, the probability of a correct response for each of the three confidence levels was calculated. On the accuracy question, probability correct was monotonically related to confidence. For Confidence Levels 1,2 , and 3 , the corresponding probabilities were $.15, .44$, and .53 . Thus, before the suggestibility manipulation there was indeed a relationship between probability correct and confidence. The picture looks very different when one examines the corresponding data on the final test (after the suggestibility manipulation). At that point, for Confidence Levels 1,2 , and 3, the corresponding probabilities were $.17, .48$, and .29 . In other words, there is no longer a simple monotonic relationship between confidence and probability correct. The manipulation apparently interfered with subjects' ability to monitor the correctness of their answers.

\section{Discussion}

Two results emerge from this experiment. First, a person who is accurate about some specific object is better able to resist a misleading suggestion about that particular object. Second, when an attempt is made to mislead a person about a detail that is patently false, the person becomes more resistant to suggestions of any kind. It is possible that the presence of a blatantly false piece of information causes a person to discount most of the rest of the information, perhaps as a result of scrutinizing it more carefully than is ordinary. If this is the mechanism by which the blatant information produces its beneficial effect, it follows that delaying the blatant information would eliminate or severely reduce its effect. Put another way, suppose subtly presented misleading information is ordinarily incorporated into a person's recollection at the time it occurs and an obviously false piece of information can prevent 
this incorporation. Then delaying the obviously false item until after incorporation is complete might lead to a rejection of the obviously false item but an acceptance of the other critical items. Alternatively, the blatantly false information may have its effects at the time a person's recollection is queried. Perhaps both the original information and a piece of subtle misinformation are retrieved from memory, but because the subtle misinformation and the blatant misinformation came from the same source, the person gives greater weight to the original information in memory. Under this hypothesis, delaying the blatant information should produce the same effect as introducing it early along with the other pieces of misinformation.

\section{EXPERIMENT 2}

\section{Method}

Subjects. Forty-five individuals completed two sessions, on 2 consecutive days, and were paid for their participation. The first session lasted approximately $1 \mathrm{~h}$, while the second session lasted nearly $2 \mathrm{~h}$.

Procedure. The subjects were told that they would see a series of slides and would then answer questions about the slides. As in Experiment 1, the overall procedure included four phases: viewing the slides, filling out an accuracy questionnaire, reading a narrative, and taking a final test. The first two phases were identical to those in Experiment 1: The slides were presented at a rate of one every $5 \mathrm{sec}$, and the accuracy test which followed it contained 30 multiple-choice items.

One day later the subjects returned for their second session. They read narratives that were somewhat different from those used in the previous study. As before, the narrative was a version of the incident that was allegedly written by a professor who had seen the slides for $30 \mathrm{sec}$ each. It contained an erroneous description of four critical items, items that were relatively peripheral to the central characters and action. However, the narrative was read in two parts. The first part was read at the beginning of the session. Following that, subjects participated in an unrelated experiment that lasted approximately $1 \mathrm{~h}$ and then they read the second part of the narrative. One-third of the subjects had the blatant misinformation about the victim's wallet placed in the first part of the narrative, one-third read the misinformation over $1 \mathrm{~h}$ later in the second portion of the narrative, and onethird read a narrative with no mention of any blatantly false information. After rating the narrative on several dimensions, such as clarity of writing, the subjects took a final test on the details of the slides.

As in Experiment 1, the final test contained 20 items, all declarative sentences with three choices for a missing word or phrase. Subjects indicated their confidence by using a 3-point scale, where 1 indicated the subject was guessing and 3 indicated high confidence. Again, they were urged to respond only on the basis of their own recollection of the slides.

\section{Results}

As before, subjects successfully resisted the blatant misinformation and responded correctly about the color of the victim's wallet. Of central interest, however, were the responses to the four critical items about which all subjects had received misinformation. As in Experiment 1, subjects who did not receive the blatant piece of misinformation were much less likely to successfully resist suggestions about the other items than
Table 1

Suggestibility Resistance on Four Critical Items for Subjects Given Immediate, Delayed, or No Blatant Misinformation

\begin{tabular}{ccc}
\multicolumn{3}{c}{ Blatant Misinformation } \\
\cline { 2 - 3 } Immediate & Delayed & None \\
\hline 4.40 & 2.80 & 2.77 \\
\hline
\end{tabular}

Note-Higher numbers indicate greater resistance.

subjects who received the blatant piece of misinformation at the same time as the other misinformation. However, when the blatant misinformation was delayed for $1 \mathrm{~h}$, subjects were no more likely to resist the subtle suggestions than were the subjects who had received no blatant misinformation at all. The data to support this statement are shown in Table 1; however, a discussion of the computation of the numbers is in order.

In Experiment 1, blatant information influenced only the ability to resist a suggestion on an item about which the subject had initially shown some accuracy. Thus, to arrive at the numbers shown in Table 1, we used only those items for which the initial response had been correct. For example, if a subject had correctly indicated that the victim's friend had been carrying a notebook, the item was included in the analysis. For all correct items, we looked at the extent to which misinformation about some property of that item (e.g., its color) was incorporated into the subject's recollection on the final test. The responses on the final test corresponding to each of the four subtle items were converted to numerical values. A score of 6 was assigned to a correct response given with high confidence. Such a score indicated very high "suggestibility resistance," in that the subject persisted in recalling a correct detail despite the presence of misinformation. A score of 1 was assigned to an incorrect response given with high confidence. Such a score, which usually indicated that the subject was highly confident in a recollection corresponding to the misinformation, indicated a very low suggestibility resistance. Mean suggestibility resistance scores were then computed for each group of subjects by taking the mean of scores provided by individual subjects in that group. The three means are displayed in Table 1.

A one-way analysis of variance performed on these data was significant $[\mathrm{F}(2,42)=19.66, \mathrm{MSe}=.67$, $\mathrm{p}<.01]$. A priori $t$ tests indicated that immediate blatant misinformation led to higher suggestibility resistance than either delayed or no blatant misinformation, and the latter two groups did not differ from one another $(\mathrm{p}<.01)$.

\section{GENERAL DISCUSSION}

Suppose a subject sees a person carrying a green notebook and later discovers that someone has called the notebook blue. Many subjects later report that they 
saw a blue notebook (see Loftus, 1977). However, if along with the information that the notebook is blue the subject also hears some blatantly false information, many fewer subjects accept the misleading blue information. In contrast, if the misleading "blue" information is introduced soon after the incident, while the blatant misinformation is delayed, that blatant information loses its power in terms of making the subject resistant to other suggestions. These results are illuminating on the issue of how new information is integrated with existing knowledge. Our results are in accord with this model: When a person experiences some event, a representation of that event is formed in memory. If the representation exists in memory when new, relevant information is introduced, the representation is updated. If the new information conflicts with what is already stored, the person either changes the old representation or leaves it as it was, depending on such things as age of the old representation, trust in the sources of the old and new information, and so on. When the conflict has been resolved, a single representation remains.

If the new information is presented along with a piece of blatantly contradictory information, the person is less likely to use the new information to update the memorial representation. It is likely that the blatant misinformation is noticed (Wilkes \& Alred, 1978), and this in turn is likely to lead to a rejection of it and other information provided by that same source. This could come about because each piece of information is better scrutinized and is consequently less likely to be used to update any prior recollection. On a later test for recollection of the original event, those given the blatant misinformation should be more accurate, since they have rejected more misinformation. The data show that they indeed are more accurate.

If the blatant information is delayed until the other misinformation has already been incorporated into the memorial representation, it is too late for subjects to cautiously scrutinize the other new information. It has already been incorporated into memory. The fact that delayed blatant misinformation does not make subjects resistant to other suggestions is inconsistent with the hypothesis that, at the time subjects are tested for their recollection, they use both the original information and the new misinformation and base their responses on the stronger of the two. This hypothesis predicts that both the delayed and the immediately presented blatant misinformation would have comparable effects. The data show that they do not.

The conclusion that new information of this sort is integrated into memory at the time it is first introduced and comprehended by a subject is supported by a number of other research efforts. For example, Cole and Loftus (in press) found that misinformation did not increase the speed with which subjects answered questions about a previously experienced event. If the original and new information produced a conflict that had to be resolved at the time of the final test, subjects should take longer to respond in the face of misinforma. tion relative to a control condition in which no new information is presented. If the new information is incorporated at the time it is introduced, prior to the final test, then subjects should not take longer. The finding that subjects do not take longer is consistent with the notion that the new information is integrated when it is introduced.

There are, of course, other interpretations of the present data, but these tend to be contradicted by prior experiments. One explanation is that the results are due simply to demand characteristics, but attempts to explore this alternative have led to the conclusion that it is unlikely (Loftus, 1979; Lof tus et al., 1978). Another possibility is that many subjects rely on their own memories very little but rather engage in an inferential task based largely on the professor's testimony. While this alternative may hold a partial germ of truth, it has difficulty accounting for the differential effects of immediate and delayed blatant misinformation.

In other laboratories, using somewhat different materials, investigators reached a conclusion similar to the one being advanced here, namely, that people integrate intervening information into a previously acquired memory representation at the time the new information is comprehended, rather than later, when a person is queried about the contents of memory (e.g., Fischhoff, 1977; Monaco, 1976; Wood, 1978). The argument has been advanced more than once that new information can cause a restructuring of cognitive representation that is quite irreversible. Any manipulation that changes the contents of memory unbeknownst to people will have profound consequences. It will render them unable to distinguish between experiences and inferences or imaginations (Harris \& Monaco, 1978; Johnson, Raye, Wang, \& Taylor, 1979). It will keep them from being critical of their inference processes, from asking questions like "How do I know a particular fact? Did I really experience it myself?" (Fischhoff, Slovic, \& Lichtenstein, 1977). It will lead sometimes to overconfidence (Lichtenstein \& Fischhoff, 1977) and sometimes to a general breakdown in ability to monitor the likelihood that knowledge is correct. Knowledge integration may be a "fundamental component of the acquisition process," important for allowing people to "put separately acquired facts together to form new ideas" (Hayes-Roth \& Thorndike, 1979, p. 91), but it is not without its costs.

\section{REFERENCES}

Cole, W. G., \& Loftus, E. F. Incorporating new information into memory. A merican Journal of Psychology, in press.

Fischnof, B. Perceived informativeness of facts. Journal of Experimental Psychology: Human Perception and Performance, 1977, 3, 349.358.

Fischroff, B., Slovic, P., \& Lichtenstein, S. Knowing with 
certainty: The appropriateness of extreme confidence. Journal of Experimental Psychology: Human Perception and Perfor. mance, $1977,3,552-564$.

Harris, R. J., \& Monaco, G. E. Psychology of pragmatic implication: Information processing between the lines. Journal of Experimental Psychology: General, 1978, 107, 1-22.

HAYES-Roth, B., \& THORNDIKE, P. W. Integration of knowledge from text. Journal of Verbal Learning and Verbal Behavior, $1979,18,91-108$

Johnson, M.J., Raye, C. L., Wang, A. Y., \& TaYlor, T. H. Fact and fantasy: The roles of accuracy and variability in confusing imaginations with perceptual experiences. Journal of Experimental Psychology: Human Learning and Memory, 1979, 5, 229-240.

Lichtenstein, S., \& Fischhoff, B. Do those who know more also know more about how much they know? Organizational Behavior and Human Performance, 1977, 20, 159-183.

LoFTus, E. F. Leading questions and the eyewitness report. Cognitive Psychology, 1975, 7, 560-572.

LofTus, E. F. Shifting human color memory. Memory \& Cognition, 1977, 5, 696-699.
Loftus, E. F. Eyewitness testimony. Cambridge: Harvard University Press, 1979.

Loftus, E. F., Mille r, D. G., \& Burns, H. J. Semantic integration of verbal information into a visual memory. Journal of Experimental Psychology: Human Learning and Memory, 1978, 4, 19-31.

Monaco, G. E. The construction of inferences as a storage phenomenon. Human Information Processing Institute Report \#76-16. Kansas State University, 1976.

Powers, P. Andriks, J. L., \& LofTus, E. F. Eyewitness accounts of females and males. Journal of Applied Psychology, 1979, 64, 339-347.

Wilkes, A. L., \& Alred, G. Prose and prejudice: Some effects of priming context on the immediate recall of information. British Journal of Psychology, 1978, 69, 123-133.

WooD, G. The knew-it-all-along effect. Journal of Experimental Psychology: Human Perception and Performance, 1978, 4, 345-353.

(Accepted for publication July 16, 1979.) 